\title{
Evaluation of Cardiovascular Disease Risk in HIV-1-Infected Patients Treated with Darunavir
}

\author{
Magda Opsomer ${ }^{1}$ - Dessislava Dimitrova ${ }^{2} \cdot$ Johan Verspeelt $^{1} \cdot$ Amy Purrington $^{3} \cdot$ Abdul Mehbob $^{4} \cdot$ Scott Chavers $^{2}$. \\ Helen $\mathrm{Pai}^{5}$. Simon Vanveggel ${ }^{1}$. Donghan $\mathrm{Luo}^{2} \cdot \mathrm{Kimberley} \mathrm{Brown}^{6} \cdot$ Christiane Moecklinghoff $^{7} \cdot$ Richard E. Nettles $^{6}$. \\ Katia Boven ${ }^{2}$
}

Published online: 10 July 2018

(c) The Author(s) 2018

\begin{abstract}
Introduction We evaluated cardiovascular disease (CVD) risk associated with darunavir treatment and examined the demographic/clinical characteristics of darunavir users based on data from Janssen-sponsored clinical trials, post-marketing pharmacovigilance databases, and administrative claims databases.

Methods First, selected CVD events [myocardial infarction, stroke, sudden death, invasive cardiovascular procedures (coronary artery angioplasty or bypass, or carotid endarterectomy)] were analyzed in 19 Janssen-sponsored phase 2-4 studies (incidence rates estimated from pooled data; 95\% confidence intervals derived from Poisson distribution). Second, analyses were conducted to identify spontaneously reported CVD events in post-marketing pharmacovigilance databases and evaluate disproportional reporting of CVD events for darunavir (using Empirical Bayesian Geometric Mean scores). Third, baseline demographic/clinical characteristics of human immunodeficiency virus-1 (HIV-1)-infected patients in general and new users of darunavir and atazanavir were explored using three US administrative claims databases.

Results Among 19 Janssen-sponsored clinical trials (treatment durations $\leq 6$ years), the CVD event rate (95\% CI) per 1000 person-years (pooled population; $n=5713$ ) was $6.15(2.91-11.89)$, and was lower for patients who used once-daily darunavir/ ritonavir 800/100 mg [0.71 (0.16-3.05); $n=1326]$ versus twice-daily darunavir/ritonavir 600/100 mg [9.21 (4.94-16.04); $n=3058]$. Trend analysis of post-marketing pharmacovigilance data showed that cumulative CVD event reporting rates for darunavir users (any dose) generally declined over time. Spontaneously reported CVD events were not disproportionately reported with darunavir versus other protease inhibitors. Compared with the general HIV-1-infected population and atazanavir users, higher proportions of darunavir users were male, older, and had comorbidities associated with CVD risk based on results from US administrative claims databases.

Conclusions This comprehensive review of Janssen-sponsored clinical trial, post-marketing, and epidemiological data does not suggest that CVD should be considered an important risk for users of darunavir.
\end{abstract}

Electronic supplementary material The online version of this article (https://doi.org/10.1007/s40268-018-0238-8) contains supplementary material, which is available to authorized users.

Magda Opsomer

mopsomer@its.jnj.com

Extended author information available on the last page of the article 


\section{Key Points}

People living with HIV-1 infection have an increased risk of developing cardiovascular disease (CVD).

Using 3 different approaches, we evaluated the CVD risk associated with use of the antiretroviral agent darunavir, and examined demographic and clinical characteristics of darunavir users.

This comprehensive review of Janssen-sponsored clinical trial, post-marketing, and epidemiological data does not suggest that CVD should be considered an important risk for users of darunavir.

Our findings are strengthened by the combination of analyses and, taken together, they offer important insights into the relationship between darunavir and CVD.

\section{Introduction}

Cardiovascular disease (CVD) is a leading cause of death in the USA and worldwide $[1,2]$ and individuals with human immunodeficiency virus (HIV)-1 infection are at increased risk of CVD [2-5]. As HIV-1-infected patients age, they are more likely than their non-infected peers to develop comorbidities such as hypertension, diabetes, dyslipidemia, and renal dysfunction $[3,5,6]$. There are multiple factors that can increase the risk of CVD in people living with HIV-1, including the HIV-1 infection itself as well as conventional CVD risk factors (e.g., tobacco use, alcohol consumption, other substance abuse, hypercholesterolemia, hypertension, elevated blood glucose, aging, male gender) [7, 8]. In HIV-1-infected individuals, continued use of antiretroviral therapy has been associated with decreased risk of fatal or nonfatal CVD events compared with episodic antiretroviral therapy (based on CD4+ cell count), although a more recent study suggests a complex relationship between antiretroviral therapy and CVD risk $[9,10]$. In the case of protease inhibitors (PIs), the use of older PIs has been associated with an increased risk of CVD-related events due to these drugs causing metabolic abnormalities such as dyslipidemia and insulin resistance; however, newer PIs have demonstrated improved CVD risk profiles [11-18].

Once-daily darunavir $800 \mathrm{mg}$, boosted by ritonavir or cobicistat and in combination with two nucleos(t)ide reverse transcriptase inhibitors, is recommended by the US Department of Health and Human Services (DHHS) as an initial antiretroviral treatment option in certain clinical situations
[17] and is also recommended by the European AIDS Clinical Society (EACS) as an initial regimen [19]. Oncedaily darunavir $800 \mathrm{mg}$ (boosted with ritonavir $100 \mathrm{mg}$ ) was approved for treatment-naive and treatment-experienced patients without darunavir resistance-associated mutations in 2009 (Europe; 2010 in the USA) [20]. Darunavir is also indicated for use in twice-daily dosing regimens in those patients with darunavir resistance-associated mutations. Twice-daily darunavir $600 \mathrm{mg}$ (boosted with ritonavir $100 \mathrm{mg}$ ) was approved for treatment-experienced, HIV-1-infected patients, including patients with triple class experience, in 2006 (USA; 2007 in Europe).

There are limited data suggesting that darunavir use is associated with increased CVD risk, although a recent observational cohort study examined the association between CVD risk and use of the contemporary PIs darunavir and atazanavir $[21,22]$, and a recent longitudinal cohort study evaluated CVD risk among 119 antiretroviral-naïve, HIV-1-infected individuals starting their first therapy [23]. In the current analyses, we evaluated the CVD risk associated with darunavir use and, in addition, examined the demographic and clinical characteristics of darunavir users in clinical trials and the real world. Assessments were based on Janssen-sponsored clinical trials, post-marketing pharmacovigilance databases, and US administrative claims databases.

\section{Methods}

\subsection{CVD Events in Janssen-Sponsored Clinical Trials}

These analyses were based on pooled data from 19 Janssen-sponsored, international, phase 2/3/4 studies of darunavir/ritonavir (summarized in Supplementary Table S1; see the electronic supplementary material) [24-41]. Patient baseline demographic and clinical characteristics, including specific risk factors for CVD, were determined for the pooled population. Medical Dictionary for Regulatory Activities (MedDRA) preferred terms corresponding to the medical concepts of CVD events (myocardial infarction, stroke, sudden death, and invasive cardiovascular procedures such as coronary artery angioplasty or bypass or carotid endarterectomy) were retrieved.

The incidence of CVD events per 1000 person-years of exposure to darunavir/ritonavir was assessed as 1000 times the number of patients with a CVD event divided by the total person-years of exposure by patients "at risk". Incidence rates overall and incidence rates over time in yearly exposure intervals for patients at risk in the pooled Janssensponsored clinical trial population were calculated for these CVD events. Results were also calculated by dosing regimen [once-daily darunavir/ritonavir 800/100 mg and twice-daily 
darunavir/ritonavir 600/100 mg (with a total daily dose of darunavir/ritonavir 1200/200 mg)], as this is an indicator of a specific target population in terms of HIV-1 and general disease characteristics. Descriptive statistics were used to calculate incidence rates and corresponding $95 \%$ confidence intervals (CIs) utilizing Poisson distribution.

\subsection{CVD Events in Post-Marketing Pharmacovigilance Databases}

Spontaneously reported, post-marketing cases of CVD events in patients treated with darunavir/ritonavir were identified in the Janssen Global Safety Database during the period from June 23, 2006 (international birth date) to December 23, 2016. A trend evaluation was conducted using the Standardized MedDRA Queries (SMQs) of central nervous system hemorrhages and cerebrovascular conditions (broad) and ischemic heart disease (broad), and the MedDRA preferred terms of carotid angioplasty, carotid artery bypass, carotid artery stent insertion, carotid artery stent removal, carotid endarterectomy, carotid revascularization, coronary angioplasty, coronary arterial stent insertion, coronary artery bypass, coronary artery stent removal, coronary brachytherapy, and coronary endarterectomy.

Empirical Bayesian Geometric Mean (EBGM) scores were derived using Multi-Item Gamma Poisson Shrinker disproportionality methodology, used to evaluate disproportionality of reporting [42] of CVD events for darunavir and other PIs (indinavir, nelfinavir, ritonavir, saquinavir, atazanavir, fosamprenavir, and tipranavir) in the US Food and Drug Administration Adverse Event Reporting System (FAERS) (2016 quarter 2 [2016Q2]) and World Health Organization VigiBase (2016Q4) databases. The threshold for disproportional reporting was $n \geq 3, \mathrm{EBGM} \geq 2$, and lower bound of the two-sided 90\% CI around EBGM $($ EB05) $>1$.

\subsection{Demographic and Clinical Characteristics of Darunavir Users in US Administrative Claims Databases}

Baseline demographic characteristics, as well as clinical characteristics (comorbidities considered to be CVD risk factors), of HIV-1-infected patients, new users of darunavir, and new users of atazanavir were explored using three US administrative claims databases [Truven Health MarketScan ${ }^{\circledR}$ Medicaid (MDCD), Truven Health MarketScan ${ }^{\circledR}$ Commercial Claims and Encounters (CCAE), and Optum Clinformatics ${ }^{\mathrm{TM}}$ Extended DataMart Socio-Economic Status (Optum); all data collected through September 2016]. Additional analyses were conducted using data from CCAE and Optum (January 2007-September 2016) to evaluate the comparability of populations, with respect to comorbidities prior to initiating darunavir and atazanavir, using propensity score matching and cohort characterization methods.

\section{Results}

\subsection{CVD Events in Janssen-Sponsored Clinical Trials}

\subsubsection{Baseline Characteristics}

A total of 5713 patients were enrolled across 19 Janssensponsored clinical trials. Baseline characteristics are summarized (Table 1). Overall, 3058 patients used twicedaily darunavir/ritonavir 600/100 mg, 1326 used oncedaily darunavir/ritonavir $800 / 100 \mathrm{mg}$, and 5713 used any darunavir/ritonavir dose (including doses other than twice-daily darunavir/ritonavir 600/100 mg and once-daily darunavir/ritonavir 800/100 mg). Among all patients (any darunavir dose), the median (range) age was 43 (18-82) years and most were male (77\%), Caucasian $(49 \%)$, and had a body mass index of $18-26 \mathrm{~kg} / \mathrm{m}^{2}(69 \%)$. Evaluation of clinical characteristics showed that compared with patients who used once-daily darunavir/ritonavir 800/100 mg, those who used twice-daily darunavir/ritonavir 600/100 mg had a higher median (range) HIV-1 RNA viral load [3.74 (1.6-6.7) vs 4.7 (1.7-7.1) $\log _{10}$ copies $/ \mathrm{mL}$, respectively] and a lower median (range) CD4+ cell count [347 (4-1888) vs $140(1-1193)$ cells $/ \mathrm{mm}^{3}$, respectively]. Other disease characteristics such as lipid levels, blood pressure, renal function, and presence of diabetes are shown in Table 1. Together, these results indicate that patients who used twice-daily darunavir/ritonavir $600 / 100 \mathrm{mg}$ tended to have more advanced HIV-1 disease and comorbidities than those who used once-daily darunavir/ritonavir $800 / 100 \mathrm{mg}$.

\subsubsection{CVD Event Incidence Rates}

The treatment duration across the 19 Janssen-sponsored clinical trials was up to 6 years (median 1.9 years; interquartile range $0.94-2.75$ years; range $0-6.1$ years). The incidence rate $(95 \% \mathrm{CI})$ per 1000 person-years of CVD events in the overall pooled population (any darunavir dose) was 6.15 (2.91-11.89), and was lower for patients using once-daily darunavir/ritonavir $800 / 100 \mathrm{mg}$ [0.71 (0.16-3.05)] versus twice-daily darunavir/ritonavir 600/100 mg [9.21 (4.94-16.04); Fig. 1]. CVD incidence rates did not increase with exposure to darunavir/ritonavir over increasing yearly intervals, and there were no CVD events in exposure intervals of more than 3 years (Fig. 1). 
Table 1 Baseline characteristics of patients enrolled in Janssen-sponsored clinical trials

\begin{tabular}{|c|c|c|c|}
\hline & $\begin{array}{l}\text { Once-daily darunavir/ } \\
\text { ritonavir } 800 / 100 \mathrm{mg} \\
(n=1326)\end{array}$ & $\begin{array}{l}\text { Twice-daily darunavir/ } \\
\text { ritonavir } 600 / 100 \mathrm{mg} \\
(n=3058)\end{array}$ & $\begin{array}{l}\text { Any darunavir/ } \\
\text { ritonavir dose* } \\
(n=5713)\end{array}$ \\
\hline \multicolumn{4}{|l|}{ Demographic characteristics } \\
\hline Age, years, median (range) & $40(18-82)$ & $43(18-78)$ & $43(18-82)$ \\
\hline \multicolumn{4}{|l|}{ Gender, $n(\%)$} \\
\hline Female & $370(28)$ & $709(23)$ & $1323(23)$ \\
\hline Male & $956(72)$ & $2349(77)$ & $4390(77)$ \\
\hline \multicolumn{4}{|l|}{ Race, $n(\%)^{\mathrm{a}}$} \\
\hline Black & $286(22)$ & $653(21)$ & $1007(18)$ \\
\hline Caucasian & $769(58)$ & $1685(55)$ & $2774(49)$ \\
\hline Hispanic & $146(11)$ & $399(13)$ & $592(10)$ \\
\hline Asian & $100(8)$ & $95(3)$ & $201(4)$ \\
\hline Other & $25(2)$ & $226(7)$ & $1139(20)$ \\
\hline \multicolumn{4}{|l|}{ BMI, $\mathrm{kg} / \mathrm{m}^{2}, n(\%)^{\mathrm{a}, \mathrm{b}}$} \\
\hline$<18$ & $20(2)$ & $162(5)$ & $236(4)$ \\
\hline $18-26$ & $888(67)$ & $2085(68)$ & $3938(69)$ \\
\hline $26.1-30$ & $277(21)$ & $515(17)$ & $1012(18)$ \\
\hline$>30$ & $141(11)$ & $295(10)$ & $499(9)$ \\
\hline \multicolumn{4}{|l|}{ Smoking status, $n(\%)^{\mathrm{a}}$} \\
\hline Smoking & $217(16)$ & $388(13)$ & $1027(18)$ \\
\hline Nonsmoking & $447(34)$ & $697(23)$ & $1581(28)$ \\
\hline Missing & $662(50)$ & $1973(65)$ & $3105(54)$ \\
\hline \multicolumn{4}{|l|}{ HIV-1 disease characteristics } \\
\hline HIV-1 RNA viral load, $\log _{10}$ copies $/ \mathrm{mL}$, median (range) ${ }^{\mathrm{c}}$ & $3.74(1.6-6.7)$ & $4.7(1.7-7.1)$ & $4.43(1.2-7.5)$ \\
\hline $\mathrm{CD} 4+$ cell count, cells $/ \mathrm{mm}^{3}$, median $(\text { range })^{\mathrm{d}}$ & $347(4-1888)$ & $140(1-1193)$ & $212(1-1888)$ \\
\hline HIV-1 transmitted by IV drug use, $n(\%)^{\mathrm{e}}$ & $54(4)$ & $209(7)$ & $520(9)$ \\
\hline \multicolumn{4}{|l|}{ Other disease characteristics } \\
\hline \multicolumn{4}{|l|}{ Lipid parameters, mg/dL, mean $(\mathrm{SE})^{\mathrm{f}}$} \\
\hline Total cholesterol & $175.79(1.43)$ & $174.1(0.855)$ & $177.07(0.665)$ \\
\hline HDL-C & $42.04(0.51)$ & $38.38(0.26)$ & $39.08(0.201)$ \\
\hline LDL-C & $97.79(1.204)$ & $96.06(0.727)$ & $98.22(0.579)$ \\
\hline Triglycerides & $152.85(3.828)$ & $249.64(4.27)$ & $226.61(2.984)$ \\
\hline \multicolumn{4}{|l|}{ Blood pressure, $\mathrm{mmHg}$, mean $(\mathrm{SE})^{\mathrm{g}}$} \\
\hline Systolic & $122.17(0.423)$ & $120.32(0.285)$ & $121.1(0.207)$ \\
\hline Diastolic & $76.64(0.288)$ & $76.03(0.199)$ & $76.55(0.143)$ \\
\hline \multicolumn{4}{|l|}{$\mathrm{eGFR}_{\mathrm{CG}}, \mathrm{mL} / \mathrm{min}, n(\%)^{\mathrm{a}, \mathrm{h}}$} \\
\hline Normal renal function $(\geq 90)$ & $871(83)$ & $2094(69)$ & $3749(72)$ \\
\hline Mild renal impairment $(\geq 60$ to $<90)$ & $165(16)$ & $782(26)$ & $1240(24)$ \\
\hline Moderate renal impairment $(\geq 30$ to $<60)$ & $15(1)$ & $172(6)$ & $236(5)$ \\
\hline Severe renal impairment $(\geq 15$ to $<30)$ & 0 & $5(<1)$ & $5(<1)$ \\
\hline Renal failure $(<15)$ & 0 & $1(<1)$ & $2(<1)$ \\
\hline Intake of lipid-lowering drugs, $n(\%)$ & $74(6)$ & $468(15)$ & $651(11)$ \\
\hline Intake of antidiabetes drugs, $n(\%)$ & $27(2)$ & $217(7)$ & $283(5)$ \\
\hline Intake of antihypertensive drugs, $n(\%)$ & $131(10)$ & $555(18)$ & $775(14)$ \\
\hline Dyslipidemia, $n(\%)^{\mathrm{i}}$ & $474(36)$ & $1942(64)$ & $3162(55)$ \\
\hline Diabetes, $n(\%)^{\mathrm{j}}$ & $42(3)$ & $285(9)$ & $415(7)$ \\
\hline Hypertension, $n(\%)^{\mathrm{k}}$ & $318(24)$ & $906(30)$ & $1499(26)$ \\
\hline
\end{tabular}

$B M I$ body mass index, $e G F R_{C G}$, estimated glomerular filtration rate calculated using the Cockcroft-Gault method, $H D L-C$ high-density lipoprotein cholesterol, $H I V-1$ human immunodeficiency virus-1, $I V$ intravenous, $L D L-C$ low-density lipoprotein cholesterol, $S E$ standard error

*Includes doses other than twice-daily darunavir/ritonavir 600/100 mg and once-daily darunavir/ritonavir 800/100 mg

${ }^{\text {a}}$ Percentages may not total $100 \%$ due to rounding 
Table 1 (continued)

${ }^{\mathrm{b}}$ Once-daily darunavir/ritonavir 800/100 mg, $n=1326$; twice-daily darunavir/ritonavir 600/100 mg, $n=3057$; any darunavir/ritonavir dose, $n=5685$

${ }^{\mathrm{c}}$ Once-daily darunavir/ritonavir 800/100 mg, $n=1311$; twice-daily darunavir/ritonavir 600/100 mg, $n=3055$; any darunavir/ritonavir dose, $n=5650$

${ }^{\mathrm{d} O n c e-d a i l y ~ d a r u n a v i r / r i t o n a v i r} 800 / 100 \mathrm{mg}, n=1325$; twice-daily darunavir/ritonavir 600/100 mg, $n=3039$; any darunavir/ritonavir dose, $n=5649$

ePercentages calculated based on the total populations, which include patients categorized as having "missing data" for HIV mode of transmission. Patients with missing data were as follows: once-daily darunavir/ritonavir 800/100 mg, $n=415$ (31\%); twice-daily darunavir/ritonavir $600 / 100 \mathrm{mg}, n=331(11 \%)$; any darunavir/ritonavir dose, $n=750(13 \%)$

${ }^{\mathrm{f}}$ Once-daily darunavir/ritonavir 800/100 mg, $n=1038$ (total cholesterol), $n=795$ (HDL-C), $n=791$ (LDL-C), $n=1038$ (triglycerides); twicedaily darunavir/ritonavir 600/100 mg, $n=3005$ (total cholesterol), $n=2727$ (HDL-C), $n=2524$ (LDL-C), $n=3005$ (triglycerides); any darunavir/ ritonavir dose, $n=5240$ (total cholesterol), $n=4625$ (HDL-C), $n=4192$ (LDL-C), $n=5258$ (triglycerides)

${ }^{\mathrm{g}}$ Once-daily darunavir/ritonavir 800/100 mg, $n=1326$ (systolic and diastolic); twice-daily darunavir/ritonavir 600/100 mg, $n=3053$ (systolic and diastolic); any darunavir/ritonavir dose, $n=5522$ (systolic), $n=5521$ (diastolic)

${ }^{\text {h} O n c e-d a i l y ~ d a r u n a v i r / r i t o n a v i r ~} 800 / 100 \mathrm{mg}, n=1051$; twice-daily darunavir/ritonavir 600/100 mg, $n=3054$; any darunavir/ritonavir dose: $n=5232$

${ }^{\mathrm{i}}$ Dyslipidemia defined as elevated total cholesterol [ $\left.>6.2 \mathrm{mmol} / \mathrm{L}(240 \mathrm{mg} / \mathrm{dL})\right]$, and/or decreased HDL-C [ $\left.<0.9 \mathrm{mmol} / \mathrm{L}(35 \mathrm{mg} / \mathrm{dL})\right]$, and/or elevated triglycerides $[>2.3 \mathrm{mmol} / \mathrm{L}(200 \mathrm{mg} / \mathrm{dL})]$

${ }_{\mathrm{j}}^{\mathrm{j}}$ Diabetes defined as fasting glucose $\geq 126 \mathrm{mg} / \mathrm{dL}(7 \mathrm{mmol} / \mathrm{L})$

${ }^{\mathrm{k}}$ Hypertension defined as elevated systolic blood pressure $(\geq 140 \mathrm{mmHg})$ and/or elevated diastolic blood pressure $(\geq 90 \mathrm{mmHg})$

A Once-daily darunavir/ritonavir $800 / 100 \mathrm{mg}$

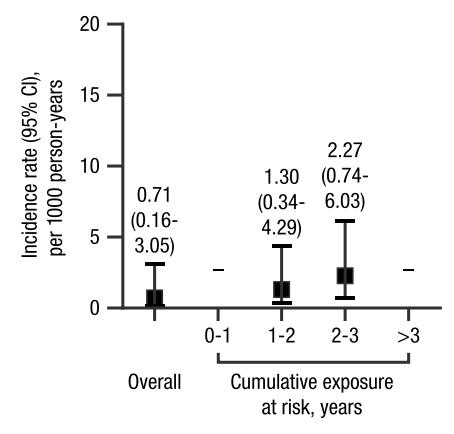

\begin{tabular}{rccccc} 
Patients, $n$ & 1326 & 1326 & 834 & 519 & 266 \\
\hline $\begin{array}{r}\text { Total CVD } \\
\text { events, } n\end{array}$ & 2 & 0 & 1 & 1 & 0 \\
\hline $\begin{array}{r}\text { Total exposure, } \\
\text { person-years }\end{array}$ & 2814 & 1207.3 & 769.7 & 440.7 & 396.1
\end{tabular}

B Twice-daily darunavir/ritonavir 600/100 mg

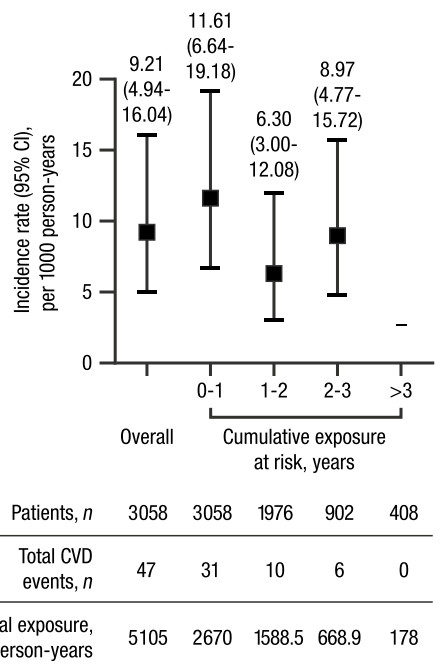

C Any darunavir/ritonavir dose*

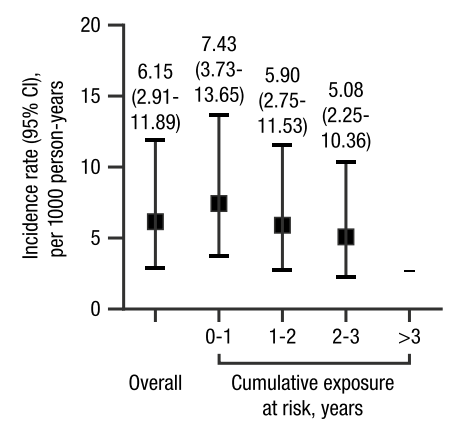

\begin{tabular}{rccccc} 
Patients, $n$ & 5713 & 5713 & 4083 & 2176 & 831 \\
\hline $\begin{array}{r}\text { Total CVD } \\
\text { events, } n\end{array}$ & 66 & 38 & 20 & 8 & 0 \\
\hline $\begin{array}{r}\text { Total exposure, } \\
\text { person-years }\end{array}$ & 10724 & 5116.1 & 3387.8 & 1573.5 & 646.6
\end{tabular}

Fig. 1 Incidence rates of CVD events overall and over time in yearly exposure intervals for patients at risk using a once-daily darunavir/ ritonavir 800/100 mg, b twice-daily darunavir/ritonavir 600/100 mg, and $\mathbf{c}$ any darunavir/ritonavir dose. $C I$ confidence interval, $C V D$ cardiovascular disease. *Includes doses other than twice-daily darunavir/ ritonavir 600/100 mg and once-daily darunavir/ritonavir 800/100 mg

\subsection{CVD Events in Post-Marketing Pharmacovigilance Databases}

Trend analysis of post-marketing pharmacovigilance data showed that cumulative reporting rates of CVD events for darunavir users generally declined over time (Fig. 2). A higher reporting rate was observed during the period of 2006-2009 (when only twice-daily darunavir/ ritonavir $600 / 100 \mathrm{mg}$ was available) compared with the period of 2010-2016 (when both twice-daily darunavir/ ritonavir 600/100 $\mathrm{mg}$ and once-daily darunavir/ritonavir $800 / 100 \mathrm{mg}$ were available). During the first period (June 23, 2006-December 23, 2006), the CVD event reporting rate per 1000 person-years was 0.348 ; through December 23,2009 , the cumulative reporting rate per 1000 personyears had decreased to 0.124; and by December 23, 2016, it was 0.042 .

Spontaneously reported CVD events were not disproportionately reported with darunavir in FAERS/VigiBase. First-generation PIs (indinavir, nelfinavir, ritonavir, 
Fig. 2 Cumulative reporting rate for spontaneous cases reporting CVD events identified with darunavir treatment by time interval (June 23, 2006December 23, 2016). CVD cardiovascular disease. *Cumulative Periodic Benefit-Risk Evaluation Report (PBRER)Periodic Safety Update Report (PSUR) reporting period

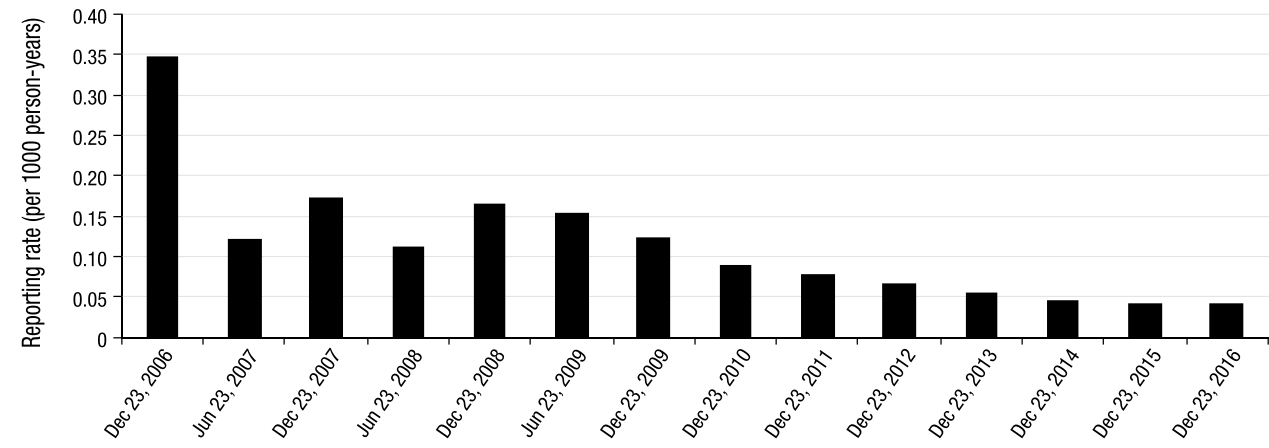

Cumulative reporting period end date (from Jun 23, 2006)*

Table 2 Signal scores from FAERS (2016Q2): cardiovascular and cerebrovascular events

\begin{tabular}{|c|c|c|c|c|c|c|c|c|c|c|c|c|}
\hline \multirow[t]{3}{*}{ Event $^{*}$} & \multicolumn{12}{|c|}{ First-generation PIs } \\
\hline & \multicolumn{3}{|c|}{ Indinavir } & \multicolumn{3}{|c|}{ Nelfinavir } & \multicolumn{3}{|c|}{ Ritonavir } & \multicolumn{3}{|c|}{ Saquinavir } \\
\hline & $n$ & EBGM & EB05 & $n$ & EBGM & EB05 & $n$ & EBGM & EB05 & $n$ & EBGM & EB05 \\
\hline Carotid artery occlusion & 1 & 0.966 & 0.213 & NR & NR & NR & 3 & 1.057 & 0.405 & 1 & 1.021 & 0.225 \\
\hline Carotid artery stenosis & 9 & 3.523 & 2.003 & 2 & 1.097 & 0.347 & 4 & 1.008 & 0.436 & 1 & 0.765 & 0.169 \\
\hline Carotid artery thrombosis & 2 & 1.535 & 0.486 & NR & NR & NR & NR & NR & NR & NR & NR & NR \\
\hline Coronary artery insufficiency & 1 & 1.23 & 0.271 & NR & NR & NR & NR & NR & NR & NR & NR & NR \\
\hline Coronary artery occlusion & 32 & 3.275 & 2.432 & 10 & 1.517 & 0.889 & 35 & 1.975 & 1.486 & 16 & 3.147 & 2.063 \\
\hline Coronary artery thrombosis & 2 & 1.367 & 0.433 & 1 & 0.636 & 0.14 & 2 & 0.635 & 0.201 & 4 & 2.846 & 1.227 \\
\hline Coronary artery stenosis & 5 & 1.959 & 0.922 & 11 & 4.151 & 2.488 & 16 & 2.405 & 1.576 & 4 & 1.998 & 0.864 \\
\hline Ischemic stroke & 1 & 0.505 & 0.111 & NR & NR & NR & 6 & 0.627 & 0.315 & NR & NR & NR \\
\hline Sudden cardiac death & NR & NR & NR & NR & NR & NR & 2 & 0.494 & 0.157 & 1 & 0.915 & 0.202 \\
\hline Ischemic heart disease (SMQ) & 373 & 1.802 & 1.653 & 253 & 1.85 & 1.667 & 494 & 1.16 & 1.077 & 177 & 1.896 & 1.673 \\
\hline Myocardial infarction (SMQ) & 325 & 1.912 & 1.744 & 229 & 2.016 & 1.806 & 430 & 1.22 & 1.126 & 161 & 2.08 & 1.824 \\
\hline $\begin{array}{l}\text { CNS hemorrhages and cerebro- } \\
\text { vascular conditions (SMQ) }\end{array}$ & 154 & 0.911 & 0.796 & 75 & 0.594 & 0.49 & 297 & 0.78 & 0.708 & 70 & 0.876 & 0.717 \\
\hline \multirow[t]{3}{*}{ Event $^{*}$} & \multicolumn{12}{|c|}{ Second-generation PIs } \\
\hline & \multicolumn{3}{|c|}{ Atazanavir } & \multicolumn{3}{|c|}{ Darunavir } & \multicolumn{3}{|c|}{ Fosamprenavir } & \multicolumn{3}{|c|}{ Tipranavir } \\
\hline & $n$ & EBGM & EB05 & $n$ & EBGM & EB05 & $n$ & EBGM & EB05 & $n$ & EBGM & EB05 \\
\hline Carotid artery occlusion & NR & NR & NR & NR & NR & NR & 1 & 1.082 & 0.238 & $\mathrm{NR}$ & NR & NR \\
\hline Carotid artery stenosis & NR & NR & NR & NR & NR & NR & NR & NR & NR & 3 & 2.829 & 1.063 \\
\hline Carotid artery thrombosis & NR & NR & NR & NR & NR & NR & NR & NR & NR & NR & NR & NR \\
\hline Coronary artery insufficiency & NR & NR & NR & NR & NR & NR & NR & NR & NR & NR & NR & NR \\
\hline Coronary artery occlusion & 5 & 0.577 & 0.272 & 6 & 1.192 & 0.598 & 2 & 0.86 & 0.272 & 1 & 0.75 & 0.165 \\
\hline Coronary artery thrombosis & 5 & 1.914 & 0.901 & NR & NR & NR & NR & NR & NR & NR & NR & NR \\
\hline Coronary artery stenosis & 10 & 2.189 & 1.282 & 4 & 1.732 & 0.749 & 3 & 1.761 & 0.675 & NR & NR & NR \\
\hline Ischemic stroke & NR & NR & NR & 3 & 0.744 & 0.285 & NR & NR & NR & NR & NR & NR \\
\hline Sudden cardiac death & 6 & 1.919 & 0.963 & 2 & 0.912 & 0.289 & NR & NR & NR & NR & NR & NR \\
\hline Ischemic heart disease (SMQ) & 176 & 0.881 & 0.777 & 176 & 0.881 & 0.777 & 176 & 0.881 & 0.777 & 8 & 0.382 & 0.21 \\
\hline Myocardial infarction (SMQ) & 149 & 0.894 & 0.78 & 149 & 0.894 & 0.78 & 149 & 0.894 & 0.78 & 6 & 0.335 & 0.168 \\
\hline $\begin{array}{l}\text { CNS hemorrhages and cerebro- } \\
\text { vascular conditions (SMQ) }\end{array}$ & 102 & 0.565 & 0.479 & 102 & 0.565 & 0.479 & 102 & 0.565 & 0.479 & 29 & 1.585 & 1.159 \\
\hline
\end{tabular}

The threshold for disproportional reporting was $n \geq 3, \mathrm{EBGM} \geq 2$, and EB05 $>1$. Events that met the disproportionality threshold are in bold 2016Q2, 2016 quarter 2, CI confidence interval, CNS central nervous system, FAERS US Food and Drug Administration Adverse Event Reporting System, EBGM Empirical Bayesian Geometric Mean, EB05 lower bound of the 2-sided 90\% CI around EBGM, MedDRA Medical Dictionary for Regulatory Activities, NR not reported, PIs protease inhibitors, SMQ Standardized MedDRA Query

*Events based on MedDRA preferred terms or SMQs (as noted) 
Table 3 Baseline characteristics of darunavir and atazanavir users and the general HIV-1-infected population in the USA

\begin{tabular}{|c|c|c|c|c|c|c|c|c|c|}
\hline \multirow[t]{2}{*}{ Parameter } & \multicolumn{3}{|l|}{ MDCD } & \multicolumn{3}{|l|}{ CCAE } & \multicolumn{3}{|l|}{ Optum } \\
\hline & $\begin{array}{l}\text { HIV-1-infected } \\
(n=80,522)\end{array}$ & $\begin{array}{l}\text { Darunavir users } \\
(n=4637)\end{array}$ & $\begin{array}{l}\text { Atazanavir } \\
\text { users }(n=4664)\end{array}$ & $\begin{array}{l}\text { HIV-1-infected } \\
(n=220,589)\end{array}$ & $\begin{array}{l}\text { Darunavir users } \\
(n=8360)\end{array}$ & $\begin{array}{l}\text { Atazanavir } \\
\text { users }(n=8977)\end{array}$ & $\begin{array}{l}\text { HIV-1-infected } \\
(n=149,145)\end{array}$ & $\begin{array}{l}\text { Darunavir users } \\
(n=4771)\end{array}$ & $\begin{array}{l}\text { Atazanavir users } \\
(n=6413)\end{array}$ \\
\hline \multicolumn{10}{|l|}{ Gender, $n(\%)$} \\
\hline Female & $36,024(45)$ & 2057 (44) & $2376(51)$ & $53,985(24)$ & $1536(18)$ & $1955(22)$ & $40,717(27)$ & 795 (17) & 1187 (19) \\
\hline Male & $44,498(55)$ & $2580(56)$ & $2288(49)$ & $166,604(76)$ & $6824(82)$ & $7022(78)$ & $108,428(73)$ & $3976(83)$ & $5226(81)$ \\
\hline \multicolumn{10}{|l|}{ Age, years } \\
\hline Mean (SD) & $41.0(14.3)$ & $42.6(11.9)$ & $40.8(12.0)$ & $41.3(11.6)$ & $44.7(10.4)$ & $42.9(10.1)$ & $42.3(13.2)$ & $47.2(11.1)$ & $43.6(10.2)$ \\
\hline$<50, n(\%)$ & $57,548(71)$ & $3211(69)$ & $3491(75)$ & $163,479(74)$ & $5424(65)$ & $6608(74)$ & $107,958(72)$ & $2757(58)$ & $4757(74)$ \\
\hline$\geq 50, n(\%)$ & $22,973(29)$ & $1426(31)$ & $1173(25)$ & $57,110(26)$ & $2936(35)$ & $2369(26)$ & $41,187(28)$ & $2014(42)$ & $1656(26)$ \\
\hline \multicolumn{10}{|l|}{ Comorbidities, \% } \\
\hline CV disorder & 30 & 64 & 52 & 23 & 50 & 38 & 29 & 61 & 44 \\
\hline Metabolic disorder & 26 & 57 & 44 & 25 & 51 & 38 & 33 & 65 & 49 \\
\hline Hypertension & 21 & 45 & 35 & 15 & 31 & 22 & 19 & 41 & 26 \\
\hline Hyperlipidemia & 10 & 27 & 18 & 17 & 37 & 26 & 25 & 50 & 37 \\
\hline Diabetes & 9 & 17 & 12 & 7 & 10 & 8 & 10 & 15 & 9 \\
\hline
\end{tabular}

$C C A E$ Truven Health MarketScan ${ }^{\circledR}$ Commercial Claims and Encounters database, $C V$ cardiovascular, $H I V-1$ human immunodeficiency virus-1, $M D C D$ Truven Health MarketScan ${ }^{\circledR}$ Medicaid database, Optum Optum Clinformatics ${ }^{\mathrm{TM}}$ Extended DataMart Socio-Economic Status database, $S D$ standard deviation

saquinavir) displayed a higher number of drug-event pairs that met the threshold for disproportionate reporting compared with second-generation PIs (atazanavir, darunavir, fosamprenavir, tipranavir) in FAERS (Table 2).

\subsection{Demographic and Clinical Characteristics of Darunavir Users in US Administrative Claims Databases}

Across the MDCD, CCAE, and Optum databases, higher proportions of darunavir users were male $(56,82$, and $83 \%$, respectively) compared with the general HIV-1-infected population $(55,76$, and $73 \%)$ and atazanavir users $(49$, 78 , and $81 \%$ ) (Table 3). Darunavir users also tended to be older than the other populations, with mean (standard deviation) ages in the three databases ranging from 42.6 (11.9) to 47.2 (11.1) years for darunavir users, 41.0 (14.3) to 42.3 (13.2) years for the general HIV-1-infected population, and 40.8 (12.0) to 43.6 (10.2) years for atazanavir users; correspondingly, higher percentages of darunavir users, versus the general HIV-1-infected population and atazanavir users, were $\geq 50$ years of age. Higher percentages of darunavir users had comorbidities considered to be CVD risk factors compared with the general HIV-1-infected population and atazanavir users. For example, the percentage of darunavir users who had a cardiovascular disorder ranged from 50 to $64 \%$ across the three databases, compared with $23-30 \%$ of the general HIV-1-infected population and $38-52 \%$ of atazanavir users. Similar results were seen for metabolic disorder, hypertension, hyperlipidemia, and diabetes.

Clinical variables that are known or are likely risk factors for CVD events were more prevalent among patients initiating darunavir versus those initiating atazanavir in the Optum database; the 13 conditions with a prevalence ratio (darunavir vs atazanavir) of $\geq 5$ are shown in Table 4 . The conditions with the highest prevalence ratio were diabetes without complication (18.550), type 2 diabetes without complication (18.219), hyperglycemia (15.017), chronic heart failure (7.589), and chronic systolic heart failure (7.214).

Further, propensity score density plots were generated from the Optum and CCAE databases to show the treatment preference between darunavir and atazanavir (Fig. 3). The plots indicate the probability of receiving darunavir or atazanavir based on a patient's clinical characteristics prior to receiving treatment. The area of overlap represents the patients in both groups who are similar with respect to clinical characteristics. Consistent results were observed in the Optum and CCAE databases, showing $<30 \%$ overlap between the two treatment groups and thus indicating that approximately $70 \%$ of patients treated with darunavir have no comparator in the atazanavir group with respect to comorbidities, drug exposures, procedures, and laboratory measures. 
Table 4 Pre-exposure conditions and cardiac risk factors

\begin{tabular}{|c|c|c|c|c|c|c|}
\hline \multirow[t]{2}{*}{ Condition } & \multicolumn{2}{|c|}{ Darunavir users } & \multicolumn{2}{|c|}{ Atazanavir users } & \multirow{2}{*}{$\begin{array}{l}\text { Absolute } \\
\text { difference }\end{array}$} & \multirow{2}{*}{$\begin{array}{l}\text { Prevalence ratio } \\
\text { (darunavir vs } \\
\text { atazanavir) }\end{array}$} \\
\hline & $n$ & Prevalence & $n$ & Prevalence & & \\
\hline Diabetes without complication & 56 & 0.011 & 4 & 0.001 & 0.140 & 18.550 \\
\hline Type 2 diabetes without complication & 55 & 0.011 & 4 & 0.001 & 0.138 & 18.219 \\
\hline Hyperglycemia & 34 & 0.007 & 3 & 0.000 & 0.106 & 15.017 \\
\hline Chronic heart failure & 63 & 0.013 & 11 & 0.002 & 0.131 & 7.589 \\
\hline Chronic systolic heart failure & 49 & 0.010 & 9 & 0.001 & 0.114 & 7.214 \\
\hline Acute systolic heart failure & 41 & 0.008 & 8 & 0.001 & 0.103 & 6.791 \\
\hline Acute heart failure & 58 & 0.012 & 12 & 0.002 & 0.121 & 6.404 \\
\hline Venous hypertension & 51 & 0.010 & 11 & 0.002 & 0.112 & 6.143 \\
\hline Dilation of aorta & 53 & 0.011 & 13 & 0.002 & 0.110 & 5.402 \\
\hline $\begin{array}{l}\text { Peripheral circulatory disorder associated with } \\
\text { type } 2 \text { diabetes }\end{array}$ & 48 & 0.010 & 12 & 0.002 & 0.104 & 5.300 \\
\hline Chronic kidney disease stage 2 & 111 & 0.022 & 28 & 0.004 & 0.159 & 5.253 \\
\hline Deep venous thrombosis of lower extremity & 84 & 0.017 & 22 & 0.003 & 0.136 & 5.059 \\
\hline Chronic kidney disease stage 1 & 57 & 0.012 & 15 & 0.002 & 0.112 & 5.035 \\
\hline
\end{tabular}

Conditions with a prevalence ratio of $\geq 5$ are reported (Optum Clinformatics ${ }^{\mathrm{TM}}$ Extended DataMart Socio-Economic Status database)

A Optum

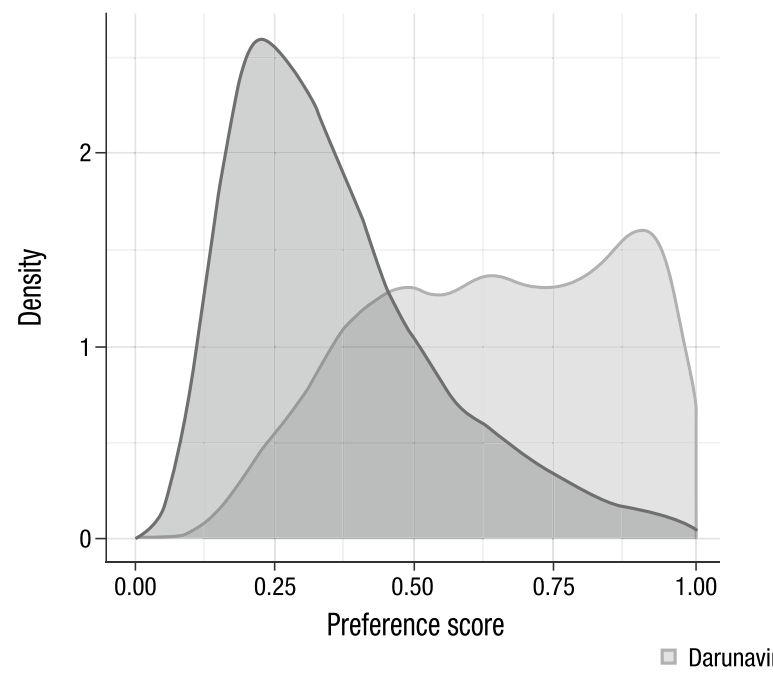

B CCAE

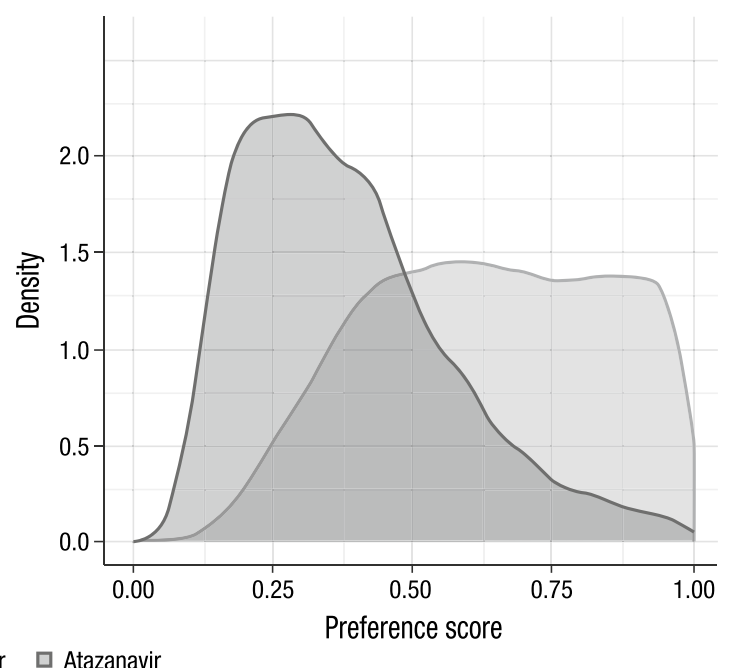

Fig. 3 Comparison of darunavir and atazanavir user baseline characteristics and comorbidities in the a Optum and b CCAE databases. $C C A E$ Truven Health MarketScan ${ }^{\circledR}$ Commercial Claims and Encoun-

\section{Discussion}

CVD is an important clinical concern for HIV-1-infected individuals because of the many factors linking these conditions; HIV-1 infection increases the risk of developing CVD, while some CVD risk factors are more common in HIV-1-infected patients relative to their peers [2-5]. While antiretroviral therapy has been associated with decreased ters database, Optum Optum Clinformatics ${ }^{\mathrm{TM}}$ Extended DataMart Socio-Economic Status database

risk of CVD events, the connection is complex $[9,10]$. Given the association between older PIs and CVD events [11-17], we examined the relationship between darunavir and CVD events using multiple approaches and datasets.

Analyses of pooled data from 19 Janssen-sponsored clinical trials did not indicate an increased risk of CVD events with exposure to darunavir/ritonavir over increasing yearly intervals (Fig. 1). Notably, the CVD event incidence rate 
was lower for patients who used once-daily darunavir/ ritonavir $800 / 100 \mathrm{mg}$ versus twice-daily darunavir/ritonavir 600/100 mg. The relatively higher CVD event rate for twice-daily darunavir/ritonavir 600/100 mg may reflect differences in the patient populations, as this dosing regimen was developed for treatment-experienced, HIV-1-infected patients with advanced disease who may be at high risk of experiencing a CVD event. This hypothesis is supported by an analysis of the baseline characteristics of these patients. Compared with patients who used once-daily darunavir/ ritonavir 800/100 mg, which included both treatment-experienced and treatment-naïve individuals, those who used twice-daily darunavir/ritonavir 600/100 mg had higher baseline HIV-1 RNA levels and lower CD4+ cell counts; moreover, higher percentages of patients who used twicedaily darunavir/ritonavir $600 / 100 \mathrm{mg}$ were being treated at baseline for, or diagnosed with, elevated lipid levels, diabetes, and hypertension. In addition to the more advanced disease and comorbidities for patients who used twice-daily darunavir/ritonavir 600/100 mg versus once-daily darunavir/ ritonavir 800/100 $\mathrm{mg}$, another possible factor leading to a higher CVD event rate with the twice-daily regimen is the higher ritonavir dose with the twice-daily versus once-daily regimen. As indicated in Table 2, as a first-generation PI, ritonavir itself may contribute to increased cardiovascular risk.

In the second set of analyses, based on real-world data, a trend analysis of post-marketing pharmacovigilance data for darunavir users showed a decline in the cumulative reporting rate of cases with CVD events from June 23, 2006 to December 23, 2016. Consistent with the pooled analysis of Janssen-sponsored clinical trials, higher CVD event reporting rates were observed during the period 2006-2009, when only twice-daily darunavir/ritonavir 600/100 mg was available, compared with the reporting rates for 2010-2016, when both twice-daily darunavir/ritonavir $600 / 100 \mathrm{mg}$ and once-daily darunavir/ritonavir $800 / 100 \mathrm{mg}$ were available. Overall, CVD event reporting rates with darunavir were significantly lower than the observed rates of CVD events in the general HIV-1-infected population [43, 44]. The data mining analysis of post-marketing pharmacovigilance databases [FAERS (2016 Q2) and VigiBase (2016 Q4)] exhibited disproportionality of CVD events mainly for first-generation PIs (e.g., indinavir, nelfinavir, ritonavir, saquinavir). Based on the results, there is no indication that use of darunavir is associated with increased reporting of CVD events over time.

The third set of analyses, an examination of baseline characteristics from three US claims databases, enabled assessment of the demographic and clinical characteristics of new users of darunavir or the contemporary PI atazanavir, as well as the general HIV-1-infected population, in the real world. These analyses demonstrated that HIV-1-infected patients who are prescribed darunavir tend to be sicker than the general HIV-1-infected population and those who are prescribed atazanavir, with more factors that could increase the risk of having a CVD event, including male gender, older age, and known CVD risk factors. The observed imbalance between populations suggests the possibility that patients with higher rates of comorbidities, and specifically cardiovascular conditions, may be channeled into darunavir treatment rather than other antiretroviral treatment. This scenario could occur if, for example, clinicians choose to treat more treatment-experienced patients or those who are medically more complex with darunavir, instead of alternative antiretroviral therapies. Darunavir was initially approved for use only in treatmentexperienced patients because of its high genetic barrier to resistance and it is often selected for individuals with uncertain adherence, who can be medically more complex than those who are highly adherent to treatment $[17,45]$. These results underscore the importance of carefully accounting for baseline characteristics when comparing outcomes between treatment groups so that confounding is minimized.

There were limitations associated with these analyses. The Janssen-sponsored clinical trials had limited durations (of up to 6 years; median duration approximately 2 years), there were gaps in data availability, few of the studies included a control arm with other PIs (precluding comparison with a reference), and the patients enrolled in these studies may not be reflective of real-world populations. The potential effect of antiretroviral therapy itself on CVD risk was also not assessed (e.g., by comparing darunavir users with untreated naive patients) [23]. Notably, a randomized study powered to definitively evaluate the association between darunavir and CVD events over time in a time-to-event analysis has not been conducted. In addition, $8 \mathrm{CVD}$ risk data were not evaluated adjusting for age and gender, which are known to be associated with CVD risk [7, 8]. In the case of the post-marketing pharmacovigilance data mining analysis, the results cannot be used to confirm or refute a causal association between drug and event; rather, these results are a measure of statistical association and need to be placed in a medical context. Moreover, underreporting of post-marketing adverse drug reactions over time, a limitation seen with a passive or voluntary reporting system, could have affected the evaluation. Results of the epidemiological analyses were based on three US claims databases and, thus, may not be generalizable to a global population. Finally, the epidemiological analysis focused only on demographics and baseline clinical characteristics, as no outcomes were analyzed. Collectively, these limitations illustrate the challenge of determining the true risk of CVD events associated with use of darunavir (and antiretroviral agents in general) [21-23]. Despite these limitations, our findings are strengthened by the combination of analyses, and taken together they offer important insights into the relationship between darunavir and CVD. 


\section{Conclusion}

Overall, this comprehensive review of clinical, post-marketing, and epidemiological data does not suggest that CVD should be considered an important risk for patients who use darunavir.

Acknowledgements The authors would like to thank Christopher Knoll, Patrick Ryan, Anthony Sena, and Eric Y. Wong for their contributions to this work.

\section{Compliance with Ethical Standards}

Research Involving Human Participants and/or Animals This article is based on previously conducted studies and does not contain any new studies with human participants or animals performed by any of the authors.

Funding Funding for this study and the article processing charges were provided by Janssen Scientific Affairs, LLC.

Medical Writing and/or Editorial Assistance Medical writing support was provided by Courtney St. Amour, PhD, of MedErgy, and was funded by Janssen Scientific Affairs, LLC.

Conflict of Interest All authors are employees of Janssen.

Authorship All authors meet the International Committee of Medical Journal Editors (ICMJE) criteria for authorship for this manuscript, take responsibility for the integrity of the work as a whole, and have given final approval for the version to be published. All authors had full access to the study data and take responsibility for the integrity of the data and accuracy of the data analysis.

Prior Presentation These data were previously presented, in part, in abstract and poster form at the 11th Annual American Conference for the Treatment of HIV (ACTHIV), April 20-22, 2017, Dallas, TX, USA.

Data Availability The datasets generated during and/or analyzed during the current study are available from the corresponding author on reasonable request.

Open Access This article is distributed under the terms of the Creative Commons Attribution-NonCommercial 4.0 International License (http://creativecommons.org/licenses/by-nc/4.0/), which permits any noncommercial use, distribution, and reproduction in any medium, provided you give appropriate credit to the original author(s) and the source, provide a link to the Creative Commons license, and indicate if changes were made.

\section{References}

1. World Health Organization. Cardiovascular diseases (CVDs): fact sheet. 2016. http://www.who.int/mediacentre/factsheets/fs317/en/. Accessed 24 March 2017.

2. US Department of Health and Human Services. Cardiovascular health. 2016. https://www.aids.gov/hiv-aids-basics/staying-healt hy-with-hiv-aids/potential-related-health-problems/cardiovasc ular-health/. Accessed March 24, 2017.

3. Schouten J, Wit FW, Stolte IG, et al. Cross-sectional comparison of the prevalence of age-associated comorbidities and their risk factors between HIV-infected and uninfected individuals: the AGEhIV cohort study. Clin Infect Dis. 2014;59(12):1787-97.

4. Palella FJ Jr, Phair JP. Cardiovascular disease in HIV infection. Curr Opin HIV AIDS. 2011;6(4):266-71.

5. Guaraldi G, Orlando G, Zona S, et al. Premature age-related comorbidities among HIV-infected persons compared with the general population. Clin Infect Dis. 2011;53(11):1120-6.

6. American Academy of HIV Medicine. The HIV and aging consensus project: recommended treatment strategies for clinicians managing older patients with HIV. 2017. https://aahivm.org/ wp-content/uploads/2017/02/Aging-report-working-documentFINAL-12.1.pdf. Accessed 13 November 2017.

7. World Heart Federation. Cardiovascular disease risk factors. 2017. http://www.world-heart-federation.org/cardiovascular-health/cardi ovascular-disease-risk-factors/. Accessed 28 April 2017.

8. American Heart Association. Coronary artery disease-coronary heart disease. 2017. http://www.heart.org/HEARTORG/Condi tions/More/MyHeartandStrokeNews/Coronary-Artery-Disease--Coronary-Heart-Disease_UCM_436416_Article.jsp\#.WQN8D fnyuUl. Accessed 28 Apr 2017.

9. Strategies for Management of Antiretroviral Therapy Study Group, El-Sadr WM, Lundgren J, et al. CD4+ count-guided interruption of antiretroviral treatment. $\mathrm{N}$ Engl J Med. 2006;355(22):2283-2296.

10. Baker JV, Sharma S, Achhra AC, et al. Changes in cardiovascular disease risk factors with immediate versus deferred antiretroviral therapy initiation among HIV-positive participants in the START (Strategic Timing of Antiretroviral Treatment) trial. J Am Heart Assoc. 2017;6(5):e004987.

11. Holstein A, Plaschke A, Egberts EH. Lipodystrophy and metabolic disorders as complication of antiretroviral therapy of HIV infection. Exp Clin Endocrinol Diabetes. 2001;109(8):389-92.

12. Holmberg SD, Moorman AC, Williamson JM, et al. Protease inhibitors and cardiovascular outcomes in patients with HIV-1. Lancet. 2002;360(9347):1747-8.

13. Calza L, Manfredi R, Chiodo F. Insulin resistance and diabetes mellitus in HIV-infected patients receiving antiretroviral therapy. Metab Syndr Relat Disord. 2004;2(4):241-50.

14. DAD Study Group, Friis-Moller N, Reiss P, et al. Class of antiretroviral drugs and the risk of myocardial infarction. N Engl J Med. 2007;356(17):1723-1735.

15. Lang S, Mary-Krause M, Cotte L, et al. Impact of individual antiretroviral drugs on the risk of myocardial infarction in human immunodeficiency virus-infected patients: a case-control study nested within the French Hospital Database on HIV ANRS cohort CO4. Arch Intern Med. 2010;170(14):1228-38.

16. Worm SW, Sabin C, Weber R, et al. Risk of myocardial infarction in patients with HIV infection exposed to specific individual antiretroviral drugs from the 3 major drug classes: the data collection on adverse events of anti-HIV drugs (D:A:D) study. J Infect Dis. 2010;201(3):318-30.

17. DHHS Panel on Antiretroviral Guidelines for Adults and Adolescents. Guidelines for the use of antiretroviral agents in adults and adolescents living with HIV. 2017. http://aidsinfo.nih.gov/ contentfiles/lvguidelines/adultandadolescentgl.pdf. Accessed 17 October 2017

18. Monforte A, Reiss P, Ryom L, et al. Atazanavir is not associated with an increased risk of cardio- or cerebrovascular disease events. AIDS. 2013;27(3):407-15.

19. European AIDS Clinical Society. EACS guidelines Version 9.0. 2017. http://www.eacsociety.org/files/guidelines_9.0-engli sh.pdf. Accessed 1 Nov 2017. 
20. Wensing AM, Calvez V, Gunthard HF, et al. 2015 Update of the drug resistance mutations in HIV-1. Top Antivir Med. 2015;23(4):132-41.

21. Ryom L, Lundgren JD, El-Sadr WM, et al. Association between cardiovascular disease \& contemporarily used protease inhibitors, oral abstract 128LB. 2017. http://www.croiconference. org/sites/default/files/uploads/croi2017-abstract-eBook.pdf. Accessed 24 March 2017.

22. Lundgren J, Mocroft A, Ryom L. Contemporary protease inhibitors and cardiovascular risk. Curr Opin Infect Dis. 2018;31(1):8-13.

23. Maggi $\mathrm{P}$, Bellacosa $\mathrm{C}$, Leone $\mathrm{A}$, et al. Cardiovascular risk in advanced naïve HIV-infected patients starting antiretroviral therapy: comparison of three different regimens-PREVALEAT II cohort. Atherosclerosis. 2017;263:398-404.

24. Currier J, Averitt Bridge D, Hagins D, et al. Sex-based outcomes of darunavir-ritonavir therapy: a single-group trial. Ann Intern Med. 2010;153(6):349-57.

25. Clumeck N, Rieger A, Banhegyi D, et al. 96 week results from the MONET trial: a randomized comparison of darunavir/ritonavir with versus without nucleoside analogues, for patients with HIV RNA $<50$ copies/mL at baseline. J Antimicrob Chemother. 2011;66(8):1878-85.

26. Arribas JR, Clumeck N, Nelson M, Hill A, van Delft Y, Moecklinghoff $\mathrm{C}$. The MONET trial: week 144 analysis of the efficacy of darunavir/ritonavir (DRV/r) monotherapy versus DRV/r plus two nucleoside reverse transcriptase inhibitors, for patients with viral load $<50$ HIV-1 RNA copies/mL at baseline. HIV Med. 2012;13(7):398-405.

27. Zorrilla CD, Wright R, Osiyemi OO, et al. Total and unbound darunavir pharmacokinetics in pregnant women infected with HIV-1: results of a study of darunavir/ritonavir 600/100 mg administered twice daily. HIV Med. 2014;15(1):50-6.

28. Crauwels HM, Kakuda TN, Ryan B, et al. Pharmacokinetics of once-daily darunavir/ritonavir in HIV-1-infected pregnant women. HIV Med. 2016;17(9):643-52.

29. Aberg JA, Tebas P, Overton ET, et al. Metabolic effects of darunavir/ritonavir versus atazanavir/ritonavir in treatment-naive, HIV type 1 -infected subjects over 48 weeks. AIDS Res Hum Retroviruses. 2012;28(10):1184-95.

30. Katlama C, Haubrich R, Lalezari J, et al. Efficacy and safety of etravirine in treatment-experienced, HIV-1 patients: pooled 48 week analysis of two randomized, controlled trials. AIDS. 2009;23(17):2289-300.

31. Katlama C, Clotet B, Mills A, et al. Efficacy and safety of etravirine at week 96 in treatment-experienced HIV type-1infected patients in the DUET-1 and DUET-2 trials. Antivir Ther. 2010;15(7):1045-52.

32. DeJesus E, Lalezari JP, Osiyemi OO, et al. Pharmacokinetics of once-daily etravirine without and with once-daily darunavir/ritonavir in antiretroviral-naive HIV type-1-infected adults. Antivir Ther. 2010;15(5):711-20.

33. Ruane PJ, Brinson C, Ramgopal M, et al. The Intelence aNd pRezista Once A Day Study (INROADS): a multicentre, single-arm, open-label study of etravirine and darunavir/ritonavir as dual therapy in HIV-1-infected early treatment-experienced subjects. HIV Med. 2015;16(5):288-96.

34. De Meyer SM, Spinosa-Guzman S, Vangeneugden TJ, de Bethune MP, Miralles GD. Efficacy of once-daily darunavir/ritonavir 800/100 mg in HIV-infected, treatment-experienced patients with no baseline resistance-associated mutations to darunavir. J Acquir Immune Defic Syndr. 2008;49(2):179-82.

35. Katlama C, Bellos N, Grinsztejn B, et al. POWER 1 and 2: combined final 144-week efficacy and safety results for darunavir/ ritonavir (DRV/r) 600/100 mg BID in treatment-experienced HIV patients. J Int AIDS Soc. 2008;11(Suppl 1):P21.

36. Molina JM, Cohen C, Katlama C, et al. Safety and efficacy of darunavir (TMC114) with low-dose ritonavir in treatment-experienced patients: 24-week results of POWER 3. J Acquir Immune Defic Syndr. 2007;46(1):24-31.

37. Orkin C, DeJesus E, Khanlou H, et al. Final 192-week efficacy and safety of once-daily darunavir/ritonavir compared with lopinavir/ritonavir in HIV-1-infected treatment-naive patients in the ARTEMIS trial. HIV Med. 2013;14(1):49-59.

38. Banhegyi D, Katlama C, da Cunha CA, et al. Week 96 efficacy, virology and safety of darunavir/r versus lopinavir/ $r$ in treatment-experienced patients in TITAN. Curr HIV Res. 2012;10(2):171-81.

39. Girard PM, Antinori A, Arribas JR, et al. Week 96 efficacy and safety of darunavir/ritonavir monotherapy vs. darunavir/ritonavir with two nucleoside reverse transcriptase inhibitors in the PROTEA trial. HIV Med. 2017;18(1):5-12.

40. Antinori A, Meraviglia P, Monforte A, et al. Effectiveness, durability, and safety of darunavir/ritonavir in HIV-1-infected patients in routine clinical practice in Italy: a postauthorization noninterventional study. Drug Des Dev Ther. 2016;10:1589-603.

41. Cahn P, Fourie J, Grinsztejn B, et al. Week 48 analysis of oncedaily vs. twice-daily darunavir/ritonavir in treatment-experienced HIV-1-infected patients. AIDS. 2011;25(7):929-39.

42. Montastruc JL, Sommet A, Bagheri H, Lapeyre-Mestre M. Benefits and strengths of the disproportionality analysis for identification of adverse drug reactions in a pharmacovigilance database. Br J Clin Pharmacol. 2011;72(6):905-8.

43. Friis-Moller N, Ryom L, Smith C, et al. An updated prediction model of the global risk of cardiovascular disease in HIV-positive persons: the Data-collection on Adverse Effects of Anti-HIV Drugs (D:A:D) study. Eur J Prev Cardiol. 2016;23(2):214-23.

44. De Socio GV, Pucci G, Baldelli F, Schillaci G. Observed versus predicted cardiovascular events and all-cause death in HIV infection: a longitudinal cohort study. BMC Infect Dis. 2017;17(1):414.

45. Lennox JL, Landovitz RJ, Ribaudo HJ, et al. Efficacy and tolerability of 3 nonnucleoside reverse transcriptase inhibitor-sparing antiretroviral regimens for treatment-naive volunteers infected with HIV-1: a randomized, controlled equivalence trial. Ann Intern Med. 2014;161(7):461-71. 


\section{Affiliations}

Magda Opsomer $^{1} \cdot$ Dessislava Dimitrova ${ }^{2} \cdot$ Johan Verspeelt $^{1} \cdot$ Amy Purrington $^{3} \cdot$ Abdul Mehbob $^{4} \cdot$ Scott Chavers $^{2}$. Helen $\mathrm{Pai}^{5} \cdot$ Simon Vanveggel ${ }^{1}$. Donghan $\mathrm{LuO}^{2} \cdot$ Kimberley Brown $^{6} \cdot$ Christiane Moecklinghoff $^{7} \cdot$ Richard E. Nettles $^{6}$. Katia Boven ${ }^{2}$

1 Janssen Research and Development, Beerse, Belgium

2 Janssen Research and Development, LLC, Titusville, NJ, USA

3 Janssen Research and Development, LLC, Horsham, PA, USA

4 Janssen-Cilag Ltd, High Wycombe, UK
5 Janssen Research and Development, LLC, Raritan, NJ, USA

6 Janssen Scientific Affairs, LLC, Titusville, NJ, USA

7 Janssen EMEA, Neuss, Germany 